\title{
Introduction to the special issue on humanoid multi-event robot athletes
}

\author{
JACKY BALTES \\ Department of Electrical Engineering, Educational Robotics Center, National Taiwan Normal University, Taipei 10610, Taiwan; \\ e-mail: jacky.baltes@ntnu.edu.tw
}

\section{Introduction}

It is with great pleasure that I present this special issue on humanoid multi-event robot athletes.

Challenge problems involving sporting events have been an important part of evaluating performance in robotics and $\mathrm{AI}$ (artificial intelligence) for more than 20 years. More recently in this period it has become a major focus for humanoid robotics, since advances in hardware and software, decreases in cost, and the goal of having robots function in domains intended to be inhabited by humans have made humanoids a more approachable tool for researchers. Consequently a variety of humanoid robot competitions have become prominent. The highest profile of these are soccer playing robots (e.g. RoboCup and AndroSot).

Multi-events are popular for human athletes. Human decathletes are widely admired for their versatility and are refereed to as the king of athletes. Modern pentathlon (running, swimming, riding, fencing, shooting) is an Olympic event modeled on the necessary skills of a military scout. Olympic Triathlon (swimming, cycling, running), biathlon (cross-country skiing and shooting), and Nordic combine (ski jumping and cross-country skiing) are multi-events with fewer events. There are also human multi-events where multiple and sometimes very distinct skill sets must be applied at different times (e.g. many human extreme endurance challenges).

While sports provide benchmarks and an important context for the public to understand work in these areas, there is also benefit in focusing on multi-events in a competition. Adaptability, flexibility, and versatility are cornerstones of human intelligence. Multi-event competitions also solve the problem of highly specialized special purpose solutions. It has been my observation that as the rules of the game become ever more complex, teams often develop special hacks (i.e. special features that work well in this but no other environment) to win. This greatly reduces the applicability of the developed solutions and leads to teams focusing on fine tuning rather than solving general issues in intelligent robotics and AI. The most obvious example is the micro-mouse competition, which was initially setup as a challenge problem for robot navigation in a maze. The maze solving algorithms have been solved years ago and nowadays the competition focuses on finding the right rubber mixture to increase the maximum acceleration and breaking of the mouse. For example, in 1998, at 2:00 am, I helped repaint all the playing fields of the RoboCup Small Sized League fields because the center-line was 1-mm wider than specified in the rules and a team complained that their vision system was unable to deal with this. This experience also taught me to never use dimensions $(5 \mathrm{~cm})$, but only ranges $(3-7 \mathrm{~cm})$ in competition rules.

Multi-events also emphasize the versatility inherent in the humanoid form: it has thus far received little attention in the research literature that across the animal kingdom, the humanoid form is not particularly suited to excellence at a single task. Humans are not the fastest runners (cheetahs), best swimmers (dolphins), or most agile climbers (monkeys). However, the humanoid form is unique in that it is near the top in a broad range of activities.

The first paper in the special issue HuroCup - Competition for Multi-Event Humanoid Robot Athletes by Baltes et al. introduce the philosophy and history behind the HuroCup competition, a nine-event multievent, where a single robot competes in sprint, obstacle run, lift and carry, basket ball, long jump, marathon, weight-lifting, wall climbing, and united soccer. The paper also describes the progress made by 
teams participating in the competition as is evidenced by improved world records. For example, the best time for the $3 \mathrm{~m}$ backward and forward sprint was reduced from 1 minute 07.50 seconds to 21.50 seconds and how in turn the organizers adapted the competition to maintain a focus on state of art research. The papers Stability Margin for Robust Walking Gaits Constructed by Center of Pressure by Tu et al. and Parameterized Gait Pattern Generator Based on LIPM With Natural ZMP References by Ho et al. focus on walking gaits for humanoid robots. The main contribution in the first is a more robust walking gait algorithm. The second paper uses an extended model of the dynamics of the legs of the robot to improve performance in walking and in kicking. Active Balancing and Turning for Alpine Skiing Robots by Iverach-Brereton et al. describes the balancing algorithm of the first skiing humanoid robot. Skiing is an interesting domain because skiis provide good stability in the lateral direction, but poor stability in the frontal plane. As researchers move from single robots to teams of collaborating robots, the ability to recognize other robots and to identify their pose becomes ever more important. This problem is addressed in Inter-Humanoid Robot Interaction with Emphasis on Detection: A Comparison Study by Shangari et al. Locomotion, especially over uneven terrain or with varying loads, is a challenging problem for humanoid robots. But navigation, that is the ability to localize the robot in the environment and to reach a target location without collisions, is also a crucial problem. Vision-Based Obstacle Avoidance System With Fuzzy Logic for Humanoid Robots by Shu et al. describe a solution for the obstacle run domain. The robot navigates through a color coded environment using a fuzzy logic controller.

\section{Acknowledgments}

The author would like to thank the reviewers for their diligent work and insightful comments which greatly improved the quality of the final product. As associate editor and as researcher, the author realizes how difficult it is nowadays to receive insightful and constructive reviews and the author really appreciates all the hard work of anonymous reviewers.

This research is supported by a grant to Jacky Baltes from the "Center of Learning Technology for Chinese" and the "Aim for the Top University Project" of the National Taiwan Normal University (NTNU) in Taipei, Taiwan. The research is also supported through a grant to Jacky Baltes by the Ministry of Education, Taiwan, and Ministry of Science and Technology, Taiwan, under Grants no. MOST 105-2218-E-003-001-MY2. 